\title{
A New Class of Generative Models for Burst-Error Characterization in Digital Wireless Channels
}

\author{
Cheng-Xiang Wang, Member, IEEE, and Wen Xu, Senior Member, IEEE
}

\begin{abstract}
Accurate and efficient generative models are significant for the design and performance evaluation of wireless communication protocols as well as error-control schemes. In this paper, deterministic processes are used to derive a new class of hard and soft generative models for simulation of digital wireless channels with hard and soft decision outputs, respectively. The proposed deterministic-process-based generative models (DPBGMs) are all based on a properly parameterized and sampled deterministic process followed by a threshold detector and two parallel mappers. The target hard and soft error sequences are provided by computer simulations of uncoded enhanced general packet radio service (EGPRS) systems with typical urban and rural area channels. Simulation results indicate that the proposed DPBGMs enable us to approximate very closely all the interested burst-error statistics of the target hard and soft error sequences. The validity of the suggested DPBGMs is further confirmed by the excellent match of the simulated frame-error rates and residual bit-error rates of coded EGPRS systems obtained from the target and generated error sequences.
\end{abstract}

Index Terms-Deterministic processes, digital wireless channels, enhanced general packet radio service (EGPRS) systems, error models, hard and soft generative models.

\section{INTRODUCTION}

W IRELESS propagation channels can roughly be classified in two major categories. The first category is analog or physical channels, where the parameters of interest are the received signal strength, the noise and/or interference power, the mobile speed, etc. Channel models for physical channels place emphasis on describing the fading characteristics of the received signal. Such models, e.g., the well-known Rayleigh and Rice models [1], are important for the design, parameter optimization, and test of the transmitter and receiver of wireless communication systems. The second category is digital channels, where we are interested in the number and distribution of error events in a sequence of bits or packets. A digital

Paper approved by C.-L. Wang, the Editor for Equalization of the IEEE Communications Society. Manuscript received July 5, 2005; revised January 12, 2006 and May 17, 2006. This paper was presented in part at the Nordic Radio Symposium, including Finnish Wireless Communications Workshop, Oulu, Finland, August 2004, and in part at the 60th IEEE Vehicular Technology Conference, Los Angeles, CA, September 2004.

C.-X. Wang is with Joint Research Institute in Signal and Image Processing, Electrical, Electronic and Computer Engineering, School of Engineering and and Physical Sciences, Heriot-Watt University, Edinburgh EH14 4AS, U.K. (e-mail: cheng-xiang.wang@hw.ac.uk).

W. $\mathrm{Xu}$ is with the Department of Electronic Engineering, Dalian University of Technology, Dalian, China, and also with the Baseband Algorithms and Standardisation Laboratory, BenQ Mobile, D-81667 Munich, Germany (e-mail: wen.xu@ieee.org).

Digital Object Identifier 10.1109/TCOMM.2007.892447 (time-discrete) channel comprises the complete transmission chain, including the transmitter, the physical channel, and the receiver in the complex baseband. Errors encountered in digital wireless channels are not independent but occur in bursts or clusters. Channel models for digital channels are called error models [2], [3], which aim at describing the statistical properties of the underlying bursty error sequences. Error models have wide applications to the design and performance evaluation of error-control schemes [3], as well as high-layer wireless communication protocols [4], [5].

Error models are either descriptive [2] or generative [3]. A descriptive model analyzes the statistical behavior of target error sequences obtained directly from a real digital channel or a computer simulation of the overall communication link. A generative model specifies a mechanism that generates error sequences statistically similar to the target error sequences [3]. Compared with a descriptive model, the main advantage of a generative model is that it can greatly reduce the computational effort for generating long error sequences, and therefore speed up simulations. In this paper, descriptive models are considered our reference models, while generative models are considered simulation models.

An error sequence can be either a hard (binary) error sequence or a soft one, depending on whether the outputs of a digital channel are based on hard decisions or soft decisions. Consequently, one can have hard generative models and soft generative models, which generate hard error sequences and soft error sequences, respectively. In the literature, five classes of hard generative models have been proposed. The first class is based on finite [3], [6]-[14] or infinite [3] state Markov chains. Gilbert [6] originally proposed a two-state Markov model. It generates in one state (good state) a hard error-free sequence, and in the other one (bad state), a sequence of errors. Elliot [7] modified Gilbert's model in such a way that errors can also occur in the good state with a small probability. The disadvantage of a two-state Markov model is its limited capability to reproduce the desired burst-error statistics. One way to overcome this problem is to enlarge the number of states. Fritchman [8] proposed Markov models with a finite number $K$ of states, which are then partitioned into two groups. One group consists of $j$ error-free states, while the other group has $K-j$ error states. Simplified Fritchman's models (SFMs) with only one error state have received wide applications [9]-[13]. For example, SFMs were applied to describe the statistical properties of high-freqency (HF) channels in [9], very (V)HF channels in [10], ultra (U)HF channels in [11], and indoor radio channels in [12] and [13]. Finite-state Markov models also include the so-called bipartite models [14]. The Markov chain used in a bipartite model 
forms a bipartite graph. Another important class of hard generative models are hidden Markov models (HMMs) [12], [13], [15], [16], which have to use a high number of HMM states in order to provide good fittings to the desired burst-error statistics. A higher state Markov model enhances the parametrization problems and makes the subsequent performance analysis of high-layer protocols increasingly difficult [5]. Furthermore, HMMs lack a direct intuition between the channel behavior and the underlying Markov chain.

Recently, three other classes of hard generative models [13], [16]-[23] were presented. The underlying error-generation mechanisms, which are completely different from Markov chains, are based on stochastic context-free grammars [13], chaos equations [16]-[19], and sum-of-sinusoids deterministic processes [20]-[23]. Stochastic context-free grammar-based hard generative models are limited to model hard error sequences having the bell-shaped error-density behavior [13]. Chaos-equation-based hard generative models [16], [17] failed to approximate some important burst-error statistics, e.g., the block-error probability distribution, with high accuracy. On the other hand, the new class of deterministic-process-based generative models (DPBGMs) [20]-[23] was demonstrated to be a promising alternative to Markov models. In particular, the DPBGM in [23] shows much better performance than the DPBGMs in [20]-[22] by accurately modeling all the interested burst-error statistics of the underlying hard error sequences. The employed target hard error sequences in [20]-[23] were obtained by computer simulations of postulated transmission systems, rather than realistic wireless communication systems. Moreover, the resulting hard error sequences generated from the developed DPBGMs in [20]-[23] were not further applied to performance simulations of error-control schemes and compared with the target hard error sequences. This implies that the applicability of the DPBGMs in [20]-[23] to the performance evaluation of coding systems was not validated.

All the above-mentioned hard generative models [3], [6]-[23] can only simulate the occurrence of binary errors. It is widely accepted that better performance of channel-coding schemes can be achieved by using soft decision decoding algorithms. In this framework, the hard generative models become useless. In the literature, only a few soft generative models were found for the simulation of digital wireless channels with soft decision outputs. They are based on either hidden Markov chains [24]-[30] or chaos equations [29], [30]. For generating soft error sequences, the HMM building becomes much more complex, since it needs to significantly increase the number of HMM states [25]. Chaos-equation-based soft generative models still result in relatively poor fittings to the desired burst-error statistics [29], [30].

The aim of this paper is twofold. First, we will follow the line of [23] and develop an improved hard generative model based on deterministic processes for realistic enhanced general packet radio service (EGPRS) systems. Uncoded EGPRS systems with hard decision outputs are adopted to provide target hard error sequences. Second, we will show that the proposed DPBGM is also capable of generating soft error sequences by slightly modifying the design procedure. In this case, uncoded EGPRS systems with soft decision outputs are used as reference transmis-

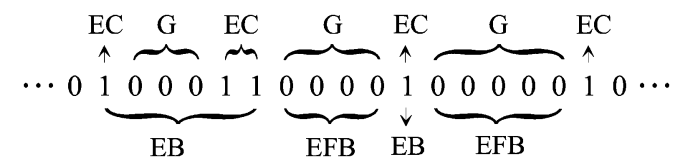

Fig. 1. Extract from a hard error sequence with $\eta=4$ as an example.

sion systems. It is shown that the proposed DPBGMs can provide excellent approximation to the desired burst-error statistics of the underlying descriptive models. The verification made by performance simulations of coded EGPRS systems with hard and soft decision decoding algorithms further confirm the reliability of the suggested models.

The paper is organized as follows. Section II briefly introduces the terms and interested burst-error statistics for both hard and soft error sequences. A general design procedure of novel hard and soft generative models based on deterministic processes is addressed in Section III. Section IV presents the adopted EGPRS systems and the resulting hard and soft error sequences. In this section, the burst-error statistics of the underlying descriptive models and the proposed generative models are also compared. Section V demonstrates the simulated frame-error rates (FERs) and residual bit-error rates (RBERs) of coded EGPRS systems obtained from the descriptive models and generative models. Finally, conclusions are drawn in Section VI.

\section{BURST-ERROR STATISTICS}

In the literature, different definitions exist for some terms describing bursty error sequences. For instance, the definition of a gap used in [9] and [10] differs from that used in [3] and [16]. For the sake of clarity, let us first introduce the terms and relevant burst-error statistics we use in this paper to characterize hard and soft error sequences. The definitions of the terms were chosen in such a way that they are convenient for the development of DPBGMs.

\section{A. Burst-Error Statistics for Hard Error Sequences}

A hard error sequence is often represented by a binary sequence of ones and zeros, with a " 1 " denoting an error bit, and a " 0 " a correctly received bit. A gap is defined as a string of consecutive zeros between two ones, having a length equal to the number of zeros [9], [10]. An error cluster is a region where the errors occur consecutively, and has a length equal to the number of ones [8]. An error-free burst is defined as an all-zero sequence with a length of at least $\eta$ bits, where $\eta$ is a positive integer [12], [14]. Compared with a gap, an error-free burst has a minimum length of $\eta$ and is not necessarily located between two errors. An error burst is a sequence of zeros and ones starting and ending with a " 1 ," and separated from neighboring error bursts by error-free bursts [12], [14]. It should be observed that the minimum length of an error burst is 1 and the number of consecutive error-free bits within an error burst is less than $\eta$. Hence, the local error density inside an error burst is greater than $1 / \eta$. To make the above concepts easily understood, we show in Fig. 1 an extract from a hard error sequence. Only in this figure, "G," "EC," "EFB," and "EB" are used to denote a gap, an error 


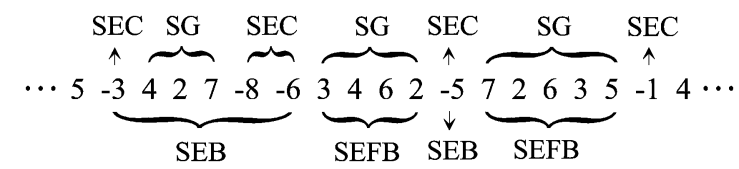

Fig. 2. Extract from a soft error sequence with $\eta=4$ as an example.

cluster, an error-free burst, and an error burst, respectively. In addition, $\eta=4$ holds as an example here.

Regarding hard error sequences, we are interested in the following burst-error statistics.

1) $G\left(m_{g}\right)$ : the gap distribution (GD), which is defined as the cumulative distribution function (CDF) of gap lengths $m_{g}$ [9].

2) $P\left(0^{m_{0}} / 1\right)$ : the error-free run distribution (EFRD), which is the probability that an error is followed by at least $m_{0}$ error-free bits [8]. The EFRD can be calculated from the GD [9]. Obviously, $P\left(0^{m_{0}} / 1\right)$ is a monotonically decreasing function of $m_{0}$, such that $P\left(0^{0} / 1\right)=1$ and $P\left(0^{m_{0}} / 1\right) \rightarrow 0$ as $m_{0} \rightarrow \infty$.

3) $P\left(1^{m_{c}} / 0\right)$ : the error cluster distribution (ECD), which is the probability that a correct bit is followed by $m_{c}$ or more consecutive bits in error [8].

4) $P_{\mathrm{EB}}\left(m_{e}\right)$ : the error burst distribution (EBD), which is the CDF of error burst lengths $m_{e}$.

5) $P_{\mathrm{EFB}}\left(m_{\bar{e}}\right)$ : the error-free burst distribution (EFBD), which is the CDF of error-free burst lengths $m_{\bar{e}}$.

6) $P(m, n)$ : the block-error probability distribution (BEPD), which is the probability that a block of $n$ bits contain at least $m$ errors. This quantity is important for determining the performance of error-correcting schemes [10].

7) $\rho(\Delta k)$ : the bit-error correlation function (BECF), which is the conditional probability that the $\Delta k$ th bit following an error bit is also in error. The BECF represents the burstiness of the channel and is useful for the design of bit interleavers [2], [3].

\section{B. Burst-Error Statistics for Soft Error Sequences}

For digital channels with $M$-bit soft decision outputs, a soft error sequence is, in general, represented by a sequence of integer numbers ranging from $-2^{M-1}$ to $2^{M-1}-1$, where $M$ is a positive integer. A negative integer indicates an error bit, while a nonnegative integer stands for a correctly received bit. The absolute value of an integer shows the reliability of the decision. Fig. 2 shows an extract from a soft error sequence. Here, $M=4$ holds as an example and therefore, the integers are located in the interval $[-8,7]$. In order to make statistical assessments of soft error sequences, some new terms and relevant burst-error statistics pertaining to soft decision outputs have to be introduced. For reasons of consistency, we will consider the following terms for soft error sequences analogous to the definitions used for hard error sequences. A soft gap (SG) is defined as a string of consecutive nonnegative integers between two negative integers, having a length equal to the number of nonnegative integers. A soft error cluster (SEC) is a region where the negative integers occur consecutively, and has a length equal to the number of negative integers. A soft error-free burst (SEFB) is defined as a sequence of nonnegative integers with a length of at least $\eta$ bits, where $\eta$ is a positive integer. Again, $\eta$ is set to be 4 as an example in Fig. 2. A soft error burst (SEB) is a sequence of integers beginning and ending with a negative integer, and separated from neighboring SEBs by SEFBs. It is important to mention that a hard error sequence can be regarded as a quantized version of a soft error sequence, i.e., $M=1$. This is also obvious by comparing Figs. 1 and 2. If we replace all the nonnegative integers by zeros and negative integers by ones in Fig. 2, then Fig. 2 will be reduced to Fig. 1.

In relevance to soft error sequences, the following burst-error statistics will be studied.

1) $G\left(m_{g}\right)$ : the soft GD (SGD), which is defined as the CDF of SG lengths $m_{g}$.

2) $P\left(m_{+}\right)$: the soft EFRD (SEFRD), which is the probability that a negative integer is followed by at least $m_{+}$nonnegative integers.

3) $P\left(m_{-}\right)$: the soft ECD (SECD), which is the probability that a nonnegative integer is followed by $m_{-}$or more negative integers.

4) $P_{\mathrm{EB}}\left(m_{e}\right)$ : the soft EBD (SEBD), which is the CDF of SEB lengths $m_{e}$.

5) $P_{\mathrm{EFB}}\left(m_{\bar{e}}\right)$ : the soft EFBD (SEFBD), which is the CDF of SEFB lengths $m_{\bar{e}}$.

6) $P(m, n)$ : the soft BEPD (SBEPD), which is the probability that a block of $n$ integers contain at least $m$ negative integers.

7) $P(S)$ : the soft decision-symbol distribution (SDSD), which is the CDF of soft decision symbols $S \in$ $\left[-2^{M-1}, 2^{M-1}-1\right]$.

It is worth stressing here that the SGD, SEFRD, SECD, SEBD, SEFBD, and SBEPD of soft error sequences will exactly be identical to the GD, EFRD, ECD, EBD, EFBD, and BEPD of hard error sequences, respectively, if the underlying hard error sequence is a corresponding quantized version of the soft error sequence. This is due to the fact that we have employed the consistent definitions of the terms and the above-mentioned burst-error statistics for hard and soft error sequences. For notational brevity, we use $G\left(m_{g}\right), P_{\mathrm{EB}}\left(m_{e}\right)$, $P_{\mathrm{EFB}}\left(m_{\bar{e}}\right)$, and $P(m, n)$ to represent (soft) GD, (soft) EBD, (soft) EFBD, and (soft) BEPD, respectively.

From the definitions of the terms, it is clear that a hard (soft) error sequence can be considered as the combination of consecutive (soft) error bursts and (soft) error-free bursts, while (soft) error bursts can further be subdivided into (soft) error clusters and (soft) gaps. To avoid a bit-by-bit processing, a hard (soft) error sequence can concisely be represented by listing the successive (soft) error-burst lengths and (soft) error-free burst lengths. This results in a (soft) error-burst recorder $\mathbf{E B}_{\text {rec }}$ and a (soft) error-free burst recorder $\mathbf{E F B} \mathbf{B}_{\text {rec }}$. Here, $\mathbf{E B}_{\text {rec }}$ is a vector which counts successive (soft) error-burst lengths, while $\mathbf{E F B}_{\text {rec }}$ records successive (soft) error-free burst lengths. Let us denote the minimum value as $m_{B 1}$ and the maximum value as $m_{B 2}$ in $\mathbf{E B}_{\text {rec }}$. This means that the lengths $m_{e}$ of (soft) error bursts satisfy $m_{B 1} \leq m_{e} \leq m_{B 2}$. By analogy, the minimum value and the maximum value in $\mathbf{E F} \mathbf{B}_{\text {rec }}$ are denoted as $m_{\bar{B} 1}$ and $m_{\bar{B} 2}$, respectively. For the convenience of developing the DPBGMs in Section III, the following quantities are defined.

1) $N_{t}$ : the total length of the target hard (soft) error sequence. 
2) $\mathcal{N}_{\mathrm{EB}}$ : the total number of (soft) error bursts, which equals the number of entries in $\mathbf{E B}_{\text {rec}}$.

3) $\mathcal{N}_{\mathrm{EFB}}$ : the total number of (soft) error-free bursts, which equals the number of entries in $\mathbf{E F B}_{\text {rec }}$.

4) $N_{\mathrm{EB}}\left(m_{e}\right)$ : the number of (soft) error bursts of length $m_{e}$ in $\mathbf{E B}_{\text {rec }}$. Apparently, $\sum_{m_{e}=m_{B 1}}^{m_{B 2}} N_{\mathrm{EB}}\left(m_{e}\right)=\mathcal{N}_{\mathrm{EB}}$ holds. The (soft) EBD $P_{\mathrm{EB}}\left(m_{e}\right)$ can then be calculated by $P_{\mathrm{EB}}\left(m_{e}\right)=\left(1 / \mathcal{N}_{\mathrm{EB}}\right) \sum_{x=m_{B 1}}^{m_{e}} N_{\mathrm{EB}}(x)$.

5) $N_{\mathrm{EFB}}\left(m_{\bar{e}}\right)$ : the number of (soft) error-free bursts of length $m_{\bar{e}}$ in $\mathbf{E F B}_{\text {rec }}$. Similarly, $\sum_{m_{\bar{e}}=m_{\bar{B} 1}}^{m_{\bar{B} 2}} N_{\mathrm{EFB}}\left(m_{\bar{e}}\right)=$ $\mathcal{N}_{\mathrm{EFB}}$ holds. The (soft) EFBD $P_{\mathrm{EFB}}\left(m_{\bar{e}}\right)$ is given by $P_{\mathrm{EFB}}\left(m_{\bar{e}}\right)=\left(1 / \mathcal{N}_{\mathrm{EFB}}\right) \sum_{x=m_{\overline{\bar{B}} 1}}^{m_{\bar{e}}} N_{\mathrm{EFB}}(x)$.

6) $\mathcal{R}_{B}$ : the ratio of the mean value $M_{\mathrm{EB}}$ of (soft) error-burst lengths to the mean value $M_{\mathrm{EFB}}$ of (soft) error-free burst lengths, i.e., $\mathcal{R}_{B}=M_{\mathrm{EB}} / M_{\mathrm{EFB}}$.

In relevance to a hard error sequence, the configuration of error-free bursts are obvious from the entries of $\mathbf{E F B}_{\text {rec }}$, since the length of an error-free burst defines the number of consecutive zeros. On the other hand, the entries of $\mathbf{E B}_{\text {rec }}$ do not provide a clear configuration of corresponding error bursts, because an error burst consists of possibly mixed zeros and ones. It is necessary to further define the following vectors.

- $\mathbf{E C G}_{i}$ : a vector which lists successive error cluster lengths and gap lengths corresponding to each entry of $\mathbf{E B}_{\text {rec. }}$. Clearly, $i=1,2, \ldots, \mathcal{N}_{\mathrm{EB}}$. Note that each vector $\mathbf{E C G}_{i}$ has an odd number of entries, with error cluster lengths as odd entries and gap lengths as even entries.

Due to the specific nature of a soft error sequence, two types of vectors need to be defined instead of the above vectors $\mathbf{E C G}_{i}$.

- $\mathbf{E B S}_{i}$ : a vector which records soft decision symbols corresponding to each entry of $\mathbf{E B}_{\text {rec }}$. Here, $i=1,2, \ldots, \mathcal{N}_{\mathrm{EB}}$. The vector $\mathbf{E B S}_{i}$ indicates the configuration of the corresponding SEB.

- $\mathbf{E F B S}_{j}$ : a vector which records soft decision symbols corresponding to each entry of $\mathbf{E F B}_{\text {rec }}$. Similarly, $j=$ $1,2, \ldots, \mathcal{N}_{\text {EFB }}$.

\section{The Novel Generative Models Based ON DETERMINISTIC PROCESSES}

It is well established that the statistics of burst errors can be estimated from the second-order statistics of fading envelope processes. This indicates the possibility of developing generative models by using fading processes. Deterministic fading processes, based on the principle of Rice's sum-of-sinusoids [31], [32], have widely been employed as physical channel simulators [1], [33], [34]. It has been shown in [20]-[23] that deterministic processes can also be used as a proper error-generation mechanism for the simulation of digital wireless channels with hard decision outputs. In this section, we will develop a general design procedure of generating hard (soft) error sequences based on deterministic processes.

It is natural to relate the generation of (soft) error bursts and (soft) error-free bursts to fading intervals and interfade intervals of a fading process, respectively. The key idea behind the proposed hard (soft) generative model is to derive directly from a deterministic process a (soft) error burst-length generator and a (soft) error-free burst-length generator. The employed deterministic process $\tilde{\zeta}(t)$ must be properly parameterized and sampled with a certain sampling interval $T_{A}$. A threshold detector with a chosen threshold $r_{\text {th }}$ then follows after the sampled deterministic process $\tilde{\zeta}\left(k T_{A}\right)$, where $k$ is a nonnegative integer. During the simulation, the level of the deterministic process will vary and cross the given threshold $r_{\text {th }}$ from time to time. If the level of $\tilde{\zeta}\left(k T_{A}\right)$ is above $r_{\mathrm{th}}$, (soft) error-free bursts are supposed to be produced at the model's output. The lengths of the generated (soft) error-free bursts equal the numbers of samples in the corresponding interfade intervals of $\tilde{\zeta}\left(k T_{A}\right)$. When the level of $\tilde{\zeta}\left(k T_{A}\right)$ falls below $r_{\text {th }}$, then (soft) error bursts will occur. The (soft) error-burst lengths equal the numbers of samples located in the corresponding fading intervals of $\tilde{\zeta}\left(k T_{A}\right)$. Consequently, a (soft) error burst-length generator $\widehat{\mathbf{E B}}_{\text {rec }}$ and a (soft) error-free burst-length generator $\widetilde{\mathbf{E F B}}_{\text {rec }}$ are obtained. Similar to the notations used for the descriptive model in Section II, we simply put the tilde sign on all affected symbols for the generative model. For example, we write $\tilde{m}_{B 1}, \tilde{\mathcal{N}}_{\mathrm{EFB}}$, and $\tilde{N}_{\mathrm{EB}}\left(m_{e}\right)$.

\section{A. The Parametrization of the Sampled Deterministic Process}

The first step for the design of the proposed hard (soft) generative model lies in the parametrization of the employed deterministic process based on the known quantities obtained from the target hard (soft) error sequence. In the following, a general idea is described to determine the parameters of the underlying deterministic process used in the hard (soft) generative model. The level-crossing rate (LCR) $\tilde{N}_{\zeta}\left(r_{\text {th }}\right)$ at the chosen threshold $r_{\text {th }}$ is fitted to the desired occurrence rate $R_{\mathrm{EB}}=\mathcal{N}_{\mathrm{EB}} / T_{t}$ of (soft) error bursts. Here, $T_{t}$ denotes the total transmission time of the reference transmission system, from which the target hard (soft) error sequence of length $N_{t}$ is obtained. The ratio $\tilde{\mathcal{R}}_{B}$ of the average duration of fades (ADF) $\tilde{T}_{\zeta_{-}}\left(r_{\mathrm{th}}\right)$ at $r_{\mathrm{th}}$ to the average duration of interfades (ADIF) $\tilde{T}_{\zeta_{+}}\left(r_{\text {th }}\right)$ at $r_{\text {th }}$ is adapted to the desired ratio $\mathcal{R}_{B}=M_{\mathrm{EB}} / M_{\mathrm{EFB}}$. Moreover, we must guarantee that the sampling interval $T_{A}$ is chosen sufficiently small in order to detect most of the level crossings and fading intervals at deep levels, i.e., $r_{\mathrm{th}} \ll 1$.

For our purpose, any forms of deterministic processes, e.g., in [1], [20]-[23], [33], and [34], with different degrees of complexities can, in principle, be used. Obviously, it is beneficial to choose a deterministic process which has as few parameters as possible in order to increase the simulation efficiency. In this paper, we will only consider the following simple continuous-time deterministic process [20]-[23]:

$$
\tilde{\zeta}(t)=\left|\tilde{\mu}_{1}(t)+j \tilde{\mu}_{2}(t)\right|
$$

where

$$
\tilde{\mu}_{i}(t)=\sum_{n=1}^{N_{i}} c_{i, n} \cos \left(2 \pi f_{i, n} t+\theta_{i, n}\right), \quad i=1,2 .
$$

In (2), $N_{i}$ defines the number of sinusoids. The phases $\theta_{i, n}$ are considered as the realizations of a random generator uniformly 
distributed over $(0,2 \pi]$. The gains $c_{i, n}$ and the discrete frequencies $f_{i, n}$ are calculated by using the method of exact Doppler spread (MEDS) [33] and are given by

$$
\begin{aligned}
c_{i, n} & =\sigma_{0} \sqrt{\frac{2}{N_{i}}} \\
f_{i, n} & =f_{\max } \sin \left[\frac{\pi}{2 N_{i}}\left(n-\frac{1}{2}\right)\right]
\end{aligned}
$$

respectively. Here, $\sigma_{0}$ is the square root of the mean power of $\tilde{\mu}_{i}(t)$, and $f_{\max }$ represents the maximum Doppler frequency. The deterministic nature of the resulting process $\tilde{\zeta}(t)$ in (1) stems from the fact that all the involved process parameters are kept constant instead of random during the simulation.

When using the MEDS with $N_{i} \geq 7$, it has been shown in [33] that the LCR $\tilde{N}_{\zeta}(r)$ of $\tilde{\zeta}(t)$ fits very closely the $\operatorname{LCR} N_{\zeta}(r)$ of a Rayleigh process, which is given by

$$
N_{\zeta}(r)=\sqrt{\frac{\beta}{2 \pi}} p_{\zeta}(r), \quad r \geq 0
$$

where

$$
\beta=2\left(\pi \sigma_{0} f_{\max }\right)^{2}
$$

and

$$
p_{\zeta}(r)=\frac{r}{\sigma_{0}^{2}} \exp \left(-\frac{r^{2}}{2 \sigma_{0}^{2}}\right), \quad r \geq 0
$$

denotes the Rayleigh distribution. It can also be shown that the ADF $\tilde{T}_{\zeta_{-}}(r)$ and the ADIF $\tilde{T}_{\zeta_{+}}(r)$ of $\tilde{\zeta}(t)$ approximate very well the corresponding quantities $T_{\zeta_{-}}(r)$ and $T_{\zeta_{+}}(r)$, respectively, of a Rayleigh process. They are given by

$$
\begin{aligned}
T_{\zeta_{-}}(r) & =\sqrt{\frac{2 \pi}{\beta}} \frac{\sigma_{0}^{2}}{r}\left[\exp \left(\frac{r^{2}}{2 \sigma_{0}^{2}}\right)-1\right], \quad r \geq 0 \\
T_{\zeta_{+}}(r) & =\sqrt{\frac{2 \pi}{\beta}} \frac{\sigma_{0}^{2}}{r}, \quad r \geq 0 .
\end{aligned}
$$

It follows that the ratio $\tilde{\mathcal{R}}_{B}$ can be expressed as

$$
\tilde{\mathcal{R}}_{B}=\frac{\tilde{T}_{\zeta_{-}}\left(r_{\mathrm{th}}\right)}{\tilde{T}_{\zeta_{+}}\left(r_{\mathrm{th}}\right)} \approx \frac{T_{\zeta_{-}}\left(r_{\mathrm{th}}\right)}{T_{\zeta_{+}}\left(r_{\mathrm{th}}\right)}=\exp \left(\frac{r_{\mathrm{th}}^{2}}{2 \sigma_{0}^{2}}\right)-1 .
$$

From the analysis above, it is clear that the second-order statistics with respect to the LCR, ADF, and ADIF of the underlying sampled deterministic process $\tilde{\zeta}\left(k T_{A}\right)$ are fully determined by the parameter vector $\Psi=\left(N_{1}, N_{2}, r_{\mathrm{th}}, \sigma_{0}, f_{\max }, T_{A}\right)$. The confronted task now is to find a proper parameter vector $\Psi$ so that the following conditions can be fulfilled: $\mathcal{R}_{B}=\left(T_{\zeta_{-}}\left(r_{\text {th }}\right) / T_{\zeta_{+}}\left(r_{\text {th }}\right)\right)$ and $R_{\mathrm{EB}}=N_{\zeta}\left(r_{\mathrm{th}}\right)$. For our purposes, it is not necessary to include all the elements of the parameter vector $\Psi$ in the design. We can first choose reasonable values for $N_{1}, N_{2}$, and $r_{\text {th }}$, e.g., $N_{1}=9, N_{2}=10$, and $r_{\text {th }}=0.09$. Then, performing
$\mathcal{R}_{B}=\left(T_{\zeta_{-}}\left(r_{\text {th }}\right) / T_{\zeta_{+}}\left(r_{\text {th }}\right)\right), \sigma_{0}$ can be calculated according to the following expression:

$$
\sigma_{0}=\frac{r_{\mathrm{th}}}{\sqrt{2 \ln \left(1+\mathcal{R}_{B}\right)}} .
$$

With the help of the relation $R_{\mathrm{EB}}=N_{\zeta}\left(r_{\mathrm{th}}\right), f_{\mathrm{max}}$ is given by

$$
f_{\max }=\frac{\mathcal{N}_{\mathrm{EB}}}{\sqrt{\pi} \sigma_{0} T_{t} p_{\zeta}\left(r_{\mathrm{th}}\right)} .
$$

The substitution of (7) into (12) yields the following explicit expression:

$$
f_{\max }=\frac{\mathcal{N}_{\mathrm{EB}}\left(1+\mathcal{R}_{B}\right)}{T_{t} \sqrt{2 \pi \ln \left(1+\mathcal{R}_{B}\right)}} .
$$

Equation (13) clearly states that $f_{\max }$ is completely determined by the known quantities $\mathcal{N}_{\mathrm{EB}}, \mathcal{R}_{B}$, and $T_{t}$ of the descriptive model, but not influenced by $r_{\text {th }}$ and $\sigma_{0}$. The sampling interval $T_{A}$ for small values of $r_{\text {th }}$ can suitably be chosen as follows [22]:

$$
T_{A} \approx \frac{4}{\sqrt{5 \pi}} T_{\zeta_{-}}\left(r_{\mathrm{th}}\right) \sqrt{-1+\sqrt{1+10 q_{s} / 3}}
$$

where $q_{s}$ is a very small quantity determining the maximum measurement error of the LCR. This implies that the probability of undetectable level crossings at $r_{\text {th }}$ is not larger than $q_{s}$. Using (8), (14) can finally be expressed as

$$
T_{A} \approx \frac{4 \sigma_{0}\left[\exp \left(\frac{r_{\mathrm{th}}^{2}}{2 \sigma_{0}^{2}}\right)-1\right]}{\sqrt{5} \pi r_{\mathrm{th}} f_{\max }} \sqrt{-1+\sqrt{1+10 q_{s} / 3}}
$$

By referring to the (11), (13), and (15), we point out that the remaining parameters $\sigma_{0}, f_{\max }$, and $T_{A}$ of $\Psi$ can all be obtained as closed-form expressions of known quantities. This allows the model users to unambiguously choose a certain set of parameters of the sampled deterministic process based on the given quantities obtained from the target hard (soft) error sequence. Consequently, the stability of the new generative model is greatly improved, compared with the model in [23]. With the resulting parameter vector $\boldsymbol{\Psi}$, a sampled deterministic process $\tilde{\zeta}\left(k T_{A}\right)$ is simulated within the necessary time interval $\left[0, \tilde{T}_{t}\right]$, i.e., $0 \leq k T_{A} \leq \tilde{T}_{t}$. Here, $\tilde{T}_{t}=T_{t} \tilde{N}_{t} / N_{t}$ with $\tilde{N}_{t}$ denoting the required length of the generated hard (soft) error sequence. The total numbers of the generated (soft) error bursts $\tilde{\mathcal{N}}_{\mathrm{EB}}$ and (soft) error-free bursts $\tilde{\mathcal{N}}_{\text {EFB }}$ can approximately be estimated from $\tilde{\mathcal{N}}_{\mathrm{EB}}=\left\lfloor\left(\tilde{N}_{t} / N_{t}\right) \mathcal{N}_{\mathrm{EB}}\right\rfloor$ and $\tilde{\mathcal{N}}_{\mathrm{EFB}}=\left\lfloor\left(\tilde{N}_{t} / N_{t}\right) \mathcal{N}_{\mathrm{EFB}}\right\rfloor$, respectively. Here, $\lfloor x\rfloor$ stands for the nearest integer to $x$ towards minus infinity, i.e., $\lfloor x\rfloor \leq x$. In this manner, a (soft) error burst length generator $\widetilde{\mathbf{E B}}_{\text {rec }}$ with $\tilde{\mathcal{N}}_{\mathrm{EB}}$ entries and a (soft) error-free burst-length generator $\widetilde{\mathbf{E F B}}_{\text {rec }}$ with $\tilde{\mathcal{N}}_{\mathrm{EFB}}$ entries are derived.

\section{B. The Mappers}

Our investigations have shown that the obtained generators $\widetilde{\mathbf{E B}}_{\text {rec }}$ and $\mathbf{E F B}_{\text {rec }}$ are in general not suitable to directly generate an acceptable (soft) EBD and (soft) EFBD, respectively. This is due to the fact that the resulting $\tilde{N}_{\mathrm{EB}}\left(m_{e}\right)$ and $\tilde{N}_{\mathrm{EFB}}\left(m_{\bar{e}}\right)$ are far from proportional to $N_{\mathrm{EB}}\left(m_{e}\right)$ and 
$N_{\mathrm{EFB}}\left(m_{\bar{e}}\right)$, respectively. Therefore, the second step of the design procedure is to develop two appropriate mappers, which map the lengths of the generated (soft) error bursts and (soft) error-free bursts to the corresponding desired lengths. The idea of the mappers is to properly modify $\widetilde{\mathbf{E B}}_{\text {rec }}$ and $\widetilde{\mathbf{E F B}}_{\text {rec }}$ such that $\tilde{N}_{\mathrm{EB}}\left(m_{e}\right)=N_{\mathrm{EB}}^{\prime}\left(m_{e}\right)$ and $\tilde{N}_{\mathrm{EFB}}\left(m_{\bar{e}}\right)_{\tilde{\mathrm{N}}}=N_{\mathrm{EFB}}^{\prime}\left(m_{\bar{e}}\right)$ hold, respectively. Here, $N_{\mathrm{EB}}^{\prime}\left(m_{e}\right)$ equals $\left\lfloor\left(\tilde{N}_{t} / N_{t}\right) N_{\mathrm{EB}}\left(m_{e}\right)\right\rfloor$ or $\left\lfloor\left(\tilde{N}_{t} / N_{t}\right) N_{\mathrm{EB}}\left(m_{e}\right)\right\rfloor+1$ for different (soft) error burst lengths $m_{e}$ in order to fulfill $\sum_{m_{e}=m_{B 1}}^{m_{B 2}} N_{\mathrm{EB}}^{\prime}\left(m_{e}\right)=\tilde{\mathcal{N}}_{\mathrm{EB}}$. Similarly, $N_{\mathrm{EFB}}^{\prime}\left(m_{\bar{e}}\right)$ equals $\left\lfloor\left(\tilde{N}_{t} / N_{t}\right) N_{\mathrm{EFB}}\left(m_{\bar{e}}\right)\right\rfloor$ or $\left\lfloor\left(\tilde{N}_{t} / N_{t}\right) N_{\mathrm{EFB}}\left(m_{\bar{e}}\right)\right\rfloor+1$ for different (soft) error-free burst lengths $m_{\bar{e}}$ to satisfy $\sum_{m_{\bar{e}}=m_{\bar{B} 1}}^{m_{\bar{B} 2}} N_{\mathrm{EFB}}^{\prime}\left(m_{\bar{e}}\right)=\tilde{\mathcal{N}}_{\mathrm{EFB}}$. Since $\tilde{N}_{\mathrm{EB}}\left(m_{e}\right)$ obtained from the modified generator $\widetilde{\mathbf{E B}}_{\text {rec }}$ after the mapping procedure is almost proportional to $N_{\mathrm{EB}}\left(m_{e}\right)$, the resulting (soft) EBD $\tilde{P}_{\mathrm{EB}}\left(m_{e}\right)$ will match well the desired (soft) EBD $P_{\mathrm{EB}}\left(m_{e}\right)$, i.e.,

$$
\begin{aligned}
\tilde{P}_{\mathrm{EB}}\left(m_{e}\right) & =\frac{1}{\tilde{\mathcal{N}}_{\mathrm{EB}}} \sum_{x=m_{B 1}}^{m_{e}} \tilde{N}_{\mathrm{EB}}(x) \\
& \approx \frac{1}{\left\lfloor\frac{\tilde{N}_{t}}{N_{t}} \mathcal{N}_{\mathrm{EB}}\right\rfloor} \sum_{x=m_{B 1}}^{m_{e}}\left\lfloor\frac{\tilde{N}_{t}}{N_{t}} N_{\mathrm{EB}}(x)\right\rfloor \\
& \approx \frac{1}{\mathcal{N}_{\mathrm{EB}}} \sum_{x=m_{B 1}}^{m_{e}} N_{\mathrm{EB}}(x)=P_{\mathrm{EB}}\left(m_{e}\right) .
\end{aligned}
$$

By analogy, the resulting (soft) EFBD $\tilde{P}_{\mathrm{EFB}}\left(m_{\bar{e}}\right)$ will be close to the desired one $P_{\mathrm{EFB}}\left(m_{\bar{e}}\right)$.

Next, we will only address how to properly modify $\widetilde{\mathbf{E B}}_{\text {rec }}$. The same idea applies also to $\widetilde{\mathbf{E F B}}_{\text {rec }}$. For each (soft) errorburst length $m_{e}\left(m_{B 1} \leq m_{e} \leq m_{B 2}\right)$, we first find the corresponding values $\ell_{m_{e}}^{1}$ and $\ell_{m_{e}}^{2}\left(\tilde{m}_{B 1} \leq \ell_{m_{e}}^{1}, \ell_{m_{e}}^{2} \leq \tilde{m}_{B 2}\right)$ in $\widetilde{\mathbf{E B}}_{\text {rec }}$ to satisfy the following conditions:

$$
\begin{aligned}
& \sum_{l=\ell_{m_{e}}^{1}}^{\ell_{m_{e}}^{2}-1} \tilde{N}_{\mathrm{EB}}(l)<N_{\mathrm{EB}}^{\prime}\left(m_{e}\right) \\
& \sum_{l=\ell_{m_{e}}^{1}}^{\ell_{m_{e}}^{2}} \tilde{N}_{\mathrm{EB}}(l) \geq N_{\mathrm{EB}}^{\prime}\left(m_{e}\right) .
\end{aligned}
$$

Let us define

$$
N_{\ell_{m_{e}}^{2}}=N_{\mathrm{EB}}^{\prime}\left(m_{e}\right)-\sum_{l=\ell_{m_{e}}^{1}}^{\ell_{m_{e}}^{2}-1} \tilde{N}_{\mathrm{EB}}(l) .
$$

Clearly, $\sum_{l=\ell_{m_{e}}^{1}}^{\ell_{m_{e}}^{2}-1} \tilde{N}_{\mathrm{EB}}(l)+N_{\ell_{m_{e}}^{2}}=N_{\mathrm{EB}}^{\prime}\left(m_{e}\right)$ holds. This indicates that if we map all (soft) error-burst lengths between $\ell_{m_{e}}^{1}$ and $\ell_{m_{e}}^{2}-1$, while only $N_{\ell_{m_{e}}^{2}}$ error-burst lengths of $\ell_{m_{e}}^{2}$ in $\widetilde{\mathbf{E B}}_{\text {rec }}$ to $m_{e}$, then $\tilde{N}_{\mathrm{EB}}\left(m_{e}\right)=N_{\mathrm{EB}}^{\prime}\left(m_{e}\right)$ will be satisfied. Note that $\ell_{m_{B 1}}^{1}=\tilde{m}_{B 1}$ and $\ell_{m_{B 2}}^{2}=\tilde{m}_{B 2}$ hold. For example, let us assume that $m_{B 1}=1, N_{\mathrm{EB}}^{\prime}(1)=5, \tilde{m}_{B 1}=10, \tilde{N}_{\mathrm{EB}}(10)=$ 2 , and $\tilde{N}_{\mathrm{EB}}(11)=3$. The application of this simple example to (17) and (18) immediately results in $\ell_{m_{e}}^{1}=10$ and $\ell_{m_{e}}^{2}=11$. Then, the error-burst lengths of 10 and 11 in $\widetilde{\mathbf{E B}}_{\text {rec }}$ are all mapped to the length 1. Consequently, $\tilde{N}_{\mathrm{EB}}(1)=N_{\mathrm{EB}}^{\prime}(1)=5$ holds. In summary, the mapper for the (soft) error burst length generator works as follows: if $l\left(\ell_{m_{e}}^{1} \leq l \leq \ell_{m_{e}}^{2}-1\right)$ samples of the deterministic process are observed in a fading interval, then a mapping $l \rightarrow m_{e}$ is first performed, and afterwards, a (soft) error burst with length $m_{e}$ is generated.

It is important to stress here that the above properly designed mappers allow the developed generative model to approximate very well any given (soft) EBD and (soft) EFBD. This makes our proposed model sufficiently general to adapt to different types of burst-error statistics.

\section{The Generation of Error Sequences}

The third step for the design procedure of the DPBGM is to generate hard (soft) error sequences from the modified generators $\widetilde{\mathbf{E B}}_{\text {rec }}$ and $\widetilde{\mathbf{E F B}}_{\text {rec }}$ after the mappers.

1) The Generation of Hard Error Sequences: The generation of error-free bursts is straightforward, since each entry of $\widetilde{\mathbf{E F B}}_{\text {rec }}$ is simply interpreted as the number of consecutive zeros. For generating error bursts, it is convenient to first construct parameter vectors $\widetilde{\mathbf{E C G}}_{j}\left(j=1,2, \ldots, \tilde{\mathcal{N}}_{\mathrm{EB}}\right)$, which reflect the configuration of each error burst in $\widetilde{\mathbf{E B}}_{\text {rec }}$ by listing the corresponding consecutive cluster lengths and gap lengths. To this end, we have to find all vectors $\mathbf{E C G}_{i}$ corresponding to error bursts with length $m_{e}$ in $\mathbf{E B}_{\text {rec }}$. Then, for all error bursts with the same length $m_{e}$ in $\mathbf{E B}_{\text {rec }}$, we randomly assign $\mathbf{E} \mathbf{C G}_{j}$ from all possible vectors $\mathbf{E C G}_{i}$. With such a vector $\widetilde{\mathbf{E C G}}_{j}$, an error burst is generated by combining consecutive error clusters (ones) and gaps (zeros). The resulting hard error sequence is simply the combination of consecutively generated error bursts and error-free bursts.

2) The Generation of Soft Error Sequences: For generating SEBs, we need first to find all vectors $\mathbf{E B S}_{i}$ corresponding to a SEB length $m_{e}$ in $\mathbf{E B}_{\text {rec. }}$. Then, we randomly choose an underlying configuration (soft decision symbols) from all possible vectors $\mathbf{E B S}_{i}$ for all SEBs with the same length $m_{e}$ in $\widetilde{\mathbf{E B}}_{\text {rec}}$. With such a vector $\mathbf{E B S}_{i}$, an SEB of length $m_{e}$ is generated. By analogy, for the generation of SEFBs, we first have to locate all vectors $\mathbf{E F B S}_{j}$ corresponding to a SEFB length $m_{\bar{e}}$ in EFB $_{\text {rec. }}$ Afterwards, the underlying configuration of a SEFB with the same length $m_{\bar{e}}$ in $\widetilde{\mathbf{E F B}}_{\text {rec }}$ is randomly selected from all possible vectors $\mathbf{E F B S}_{j}$. In this manner, a SEFB of length $m_{\bar{e}}$ is produced. The resulting soft error sequence is simply the combination of consecutively generated SEBs and SEFBs.

In short, the design procedure of the proposed DPBGM involves three steps: the parametrization of the sampled deterministic process, the development of two mappers, and the generation of hard (soft) error sequences. We call the first two steps the simulation setup phase, and the last step the simulation run phase. It should be noted that, although the simulation setup phase of the proposed DPBGM requires relatively long time, the simulation run phase is very fast, since it determines directly (soft) error burst and (soft) error-free burst lengths instead of bit sequences. The design procedures of a hard generative model and a soft generative model based on deterministic processes differ mainly in the last step. The general block diagram of the proposed DPBGM is depicted in Fig. 3. 


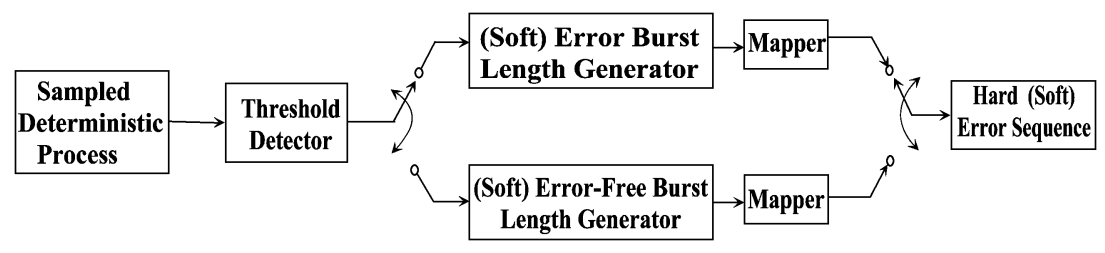

Fig. 3. General block diagram of the proposed DPBGM.

\section{Simulation RESUlts AND Discussions}

In this section, the performance of the novel generative models is investigated by applying the mechanism to experimental error sequences. The burst-error statistics defined in Section II are used here as the performance criteria. In general, one generative model outperforms another if it better fits the important statistics, e.g., the (soft) BEPD, of the descriptive model.

Uncoded EGPRS transmission systems were employed to generate target soft error sequences. The underlying digital channels are composed of a Gaussian minimum-shift keying (GMSK) modulator, a propagation channel with cochannel interference, a GMSK demodulator, and a 4-bit soft-decision Viterbi equalizer. The data were transmitted in time-division multiple-access (TDMA) bursts of 116 bits with a transmission rate of $F_{s}=270.8 \mathrm{~kb} / \mathrm{s}$. As specified in [35], the deployed propagation channels can be denoted as NAMEx. Here, $x$ represents the vehicle speed in $\mathrm{km} / \mathrm{h}$ and NAME stands for the name of a particular channel, e.g., a typical urban (TU) channel and a rural area (RA) channel. Also, the system can use either no frequency-hopping (NFH), or ideal FH (IFH), which implies perfect decorrelation between TDMA bursts [35]. In this paper, we have considered one typical narrowband propagation channel profile, RA $275 \mathrm{NFH}$, as well as three typical wideband propagation channel profiles: TU3 IFH, TU3 NFH, TU50 NFH [35]. In the case of $\mathrm{NFH}$, since data are transmitted using a GSM carrier within a bandwidth of $200 \mathrm{kHz}$, the system is a narrowband one. When FH is employed, the system operates in a wide frequency band. Depending on the actual frequency band assigned to the operator, a bandwidth of $5 \mathrm{MHz}$ or more is, in fact, used.

The target soft error sequences of length $N_{t}=15 \times 10^{6}$ were produced at carrier-to-interference ratios (CIRs) of 5, 7, 8, 9, $11,13,15$, and $17 \mathrm{~dB}$. The total transmission time is therefore $T_{t}=N_{t} / F_{s}=55.3914 \mathrm{~s}$. The target hard error sequences were obtained as quantized versions of the corresponding soft error sequences. This is the same as we obtain target hard error sequences from the uncoded EGPRS systems with a hard decision Viterbi equalizer. By using the design procedure of the proposed DPBGMs in Section III, hard (soft) error sequences of length $\tilde{N}_{t}=20 \times 10^{6}$ were generated. It follows that the necessary simulation time of the deterministic processes is $\tilde{T}_{t}=T_{t} \tilde{N}_{t} / N_{t}=$ $73.8552 \mathrm{~s}$. For the sake of brevity, only the simulation results of the EGPRS system with the TU3 IFH channel will be presented here. For other channel types, the presented error models perform similarly well.

Let us first study the performance of the obtained hard generative model in terms of the interested burst-error statistics. The GDs, the EFRDs, the ECDs, the EBDs, and EFBDs with

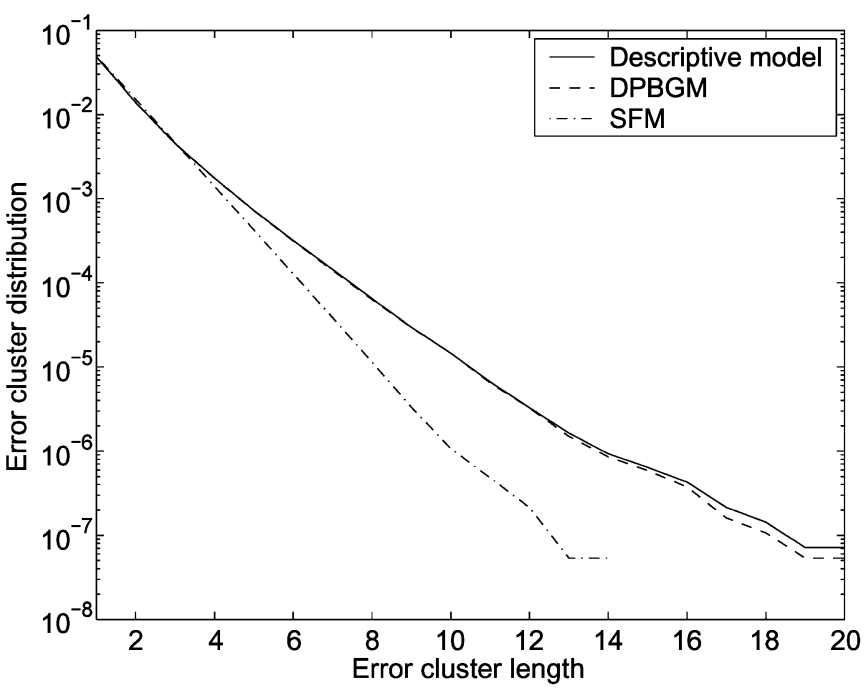

Fig. 4. ECDs of the descriptive model and the hard generative models.

$\eta=800$, the BEPDs with blocks of 116 bits $(n=116)$ per TDMA burst, and the BECFs calculated from the target and generated hard error sequences were compared. For further comparison purposes, the relevant results of a six-state SFM were also presented. The transition probability matrix of a $K$-state SFM is calculated by expressing the EFRD $P\left(0^{m_{0}} / 1\right)$ as the sum of $K-1$ exponentials with suitable weighting coefficients [8]. This procedure has to involve curve fitting techniques and is called the simulation setup phase of an SFM. From the transition probability matrix of a SFM, hard error sequences can be generated with any desired length, which is considered as the simulation run phase of an SFM. In our case, the fitting of $P\left(0^{m_{0}} / 1\right)$ is achieved by using five exponentials. Our experiments have shown that no better performance can be obtained from SFMs with more than six states. The same conclusion was also given in [20]-[23].

As an example, we will only show the simulation results of the descriptive model and two hard generative models for the EGPRS system with the TU3 IFH channel at a CIR of $8 \mathrm{~dB}$. The chosen parameter vector for the underlying sampled deterministic process was $\Psi=(9,10,0.09,0.0783,73.22 \mathrm{~Hz}, 0.8132 \mathrm{~ms})$, which were calculated from the given quantities $\mathcal{R}_{B}=0.9344$, $\mathcal{N}_{\mathrm{EB}}=4269$, and $q_{s}=0.01$. Figs. $4-7$ show the resulting ECDs, EBDs, BEPDs, and BECFs of the descriptive model and both hard generative models, respectively. The results for the GDs, EFRDs, and EFBDs of the three models are not presented here since they are very close to each other. As expected, all these curves for the DPBGM have very excellent agreements with the target ones. However, relatively large deviations were 


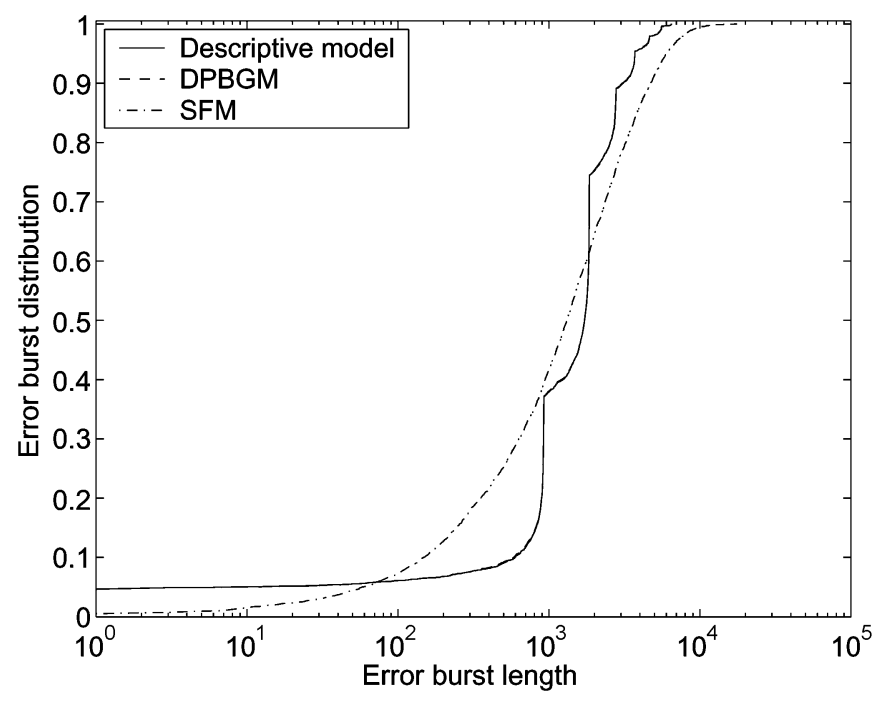

Fig. 5. EBDs of the descriptive model and the hard generative models.

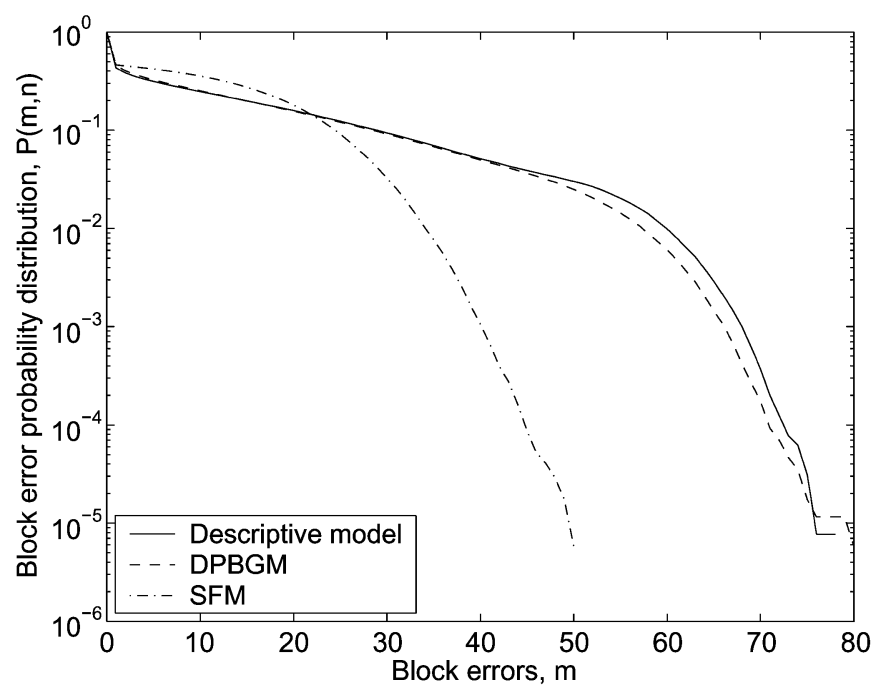

Fig. 6. BEPDs of the descriptive model and the hard generative models.

found for the fittings to the desired ECD, EBD, BEPD, and BECF by using the SFM. This demonstrates that the SFM fails to model some characteristics, especially the correlation properties, of the target hard error sequence. Concerning the simulation time, we do not need to compare the simulation setup phase of the DPBGM and the SFM. For generating a hard error sequence of length $20 \times 10^{6}$, the DPBGM and the SFM need for their simulation run phase about 1.25 and $107.5 \mathrm{~s}$, respectively. Hence, from both the accuracy and simulation efficiency points of view, the superiority of the DPBGM over the SFM is obvious. Experiments have shown that the descriptive model needs approximately $8.5 \mathrm{~h}$ for generating a hard error sequence of length $20 \times 10^{6}$. This clearly indicates the advantage of the generative models over the underlying descriptive model.

Then, we investigate the interested burst-error statistics of the proposed soft generative model. As mentioned in Section II, the SGDs, SEFRDs, SECDs, SEBDs, SEFBDs, and SBEPDs of target soft error sequences are exactly identical

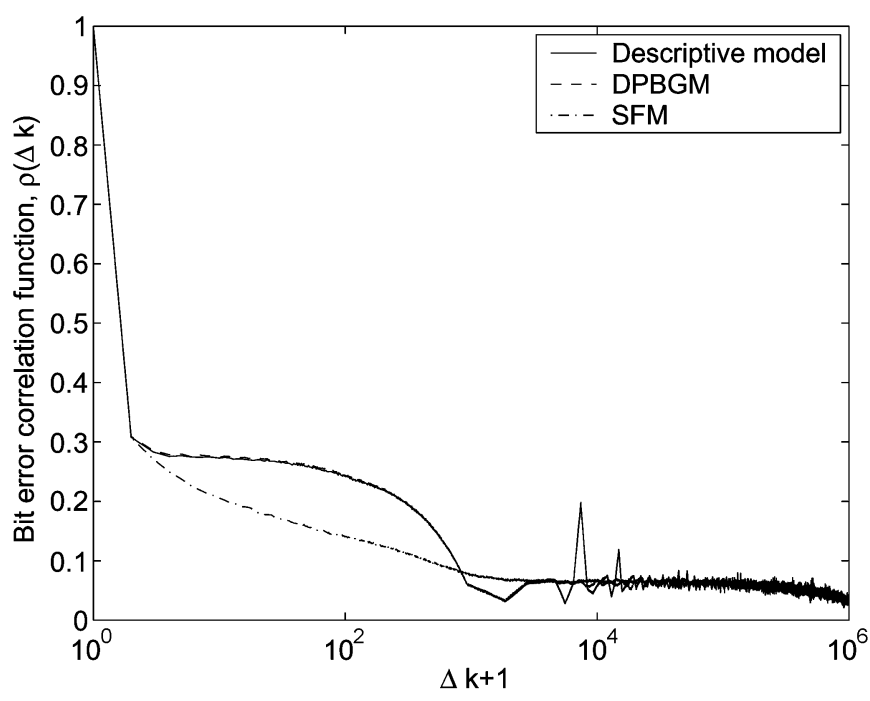

Fig. 7. BECFs of the descriptive model and the hard generative models.

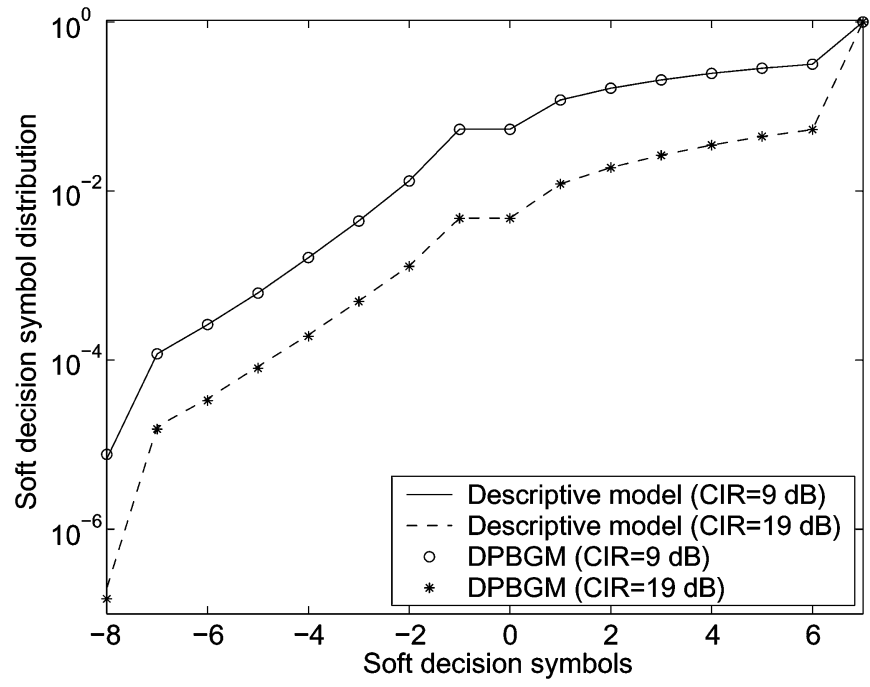

Fig. 8. SDSDs of the descriptive model and the DPBGM.

to the corresponding GDs, EFRDs, ECDs, EBDs, EFBDs, and BEPDs, respectively, of target hard error sequences. This is due to the fact that the target hard error sequences were obtained as quantized versions of the target soft error sequences. By using the proposed soft DPBGM, the resulting SGD, SEFRD, SECD, SEBD, SEFBD, and SBEPD are the same as those obtained from the hard generative model. Therefore, these statistics have excellent approximations to those of the descriptive model, as we have verified for the hard generative model. For brevity of presentation, the results are omitted here. Fig. 8 demonstrates the good match between the SDSDs of the descriptive model and the DPBGM. As examples, the CIRs of 9 and $19 \mathrm{~dB}$ were selected. In the case of CIR $=9 \mathrm{~dB}$, the ratio $\mathcal{R}_{B}=0.73865$ and $\mathcal{N}_{\mathrm{EB}}=4263$ SEBs were obtained. With $q_{s}=0.01$, the chosen parameter vector for the corresponding deterministic process was $\Psi=$ $(9,10,0.09,0.0856,71.785 \mathrm{~Hz}, 0.71593 \mathrm{~ms})$. For CIR $=19 \mathrm{~dB}$, $\mathcal{R}_{B}=0.093488$ and $\mathcal{N}_{\mathrm{EB}}=2006$ hold. The chosen parameter vector was $\Psi=(9,10,0.09,0.21288,52.841 \mathrm{~Hz}, 0.30635 \mathrm{~ms})$. 


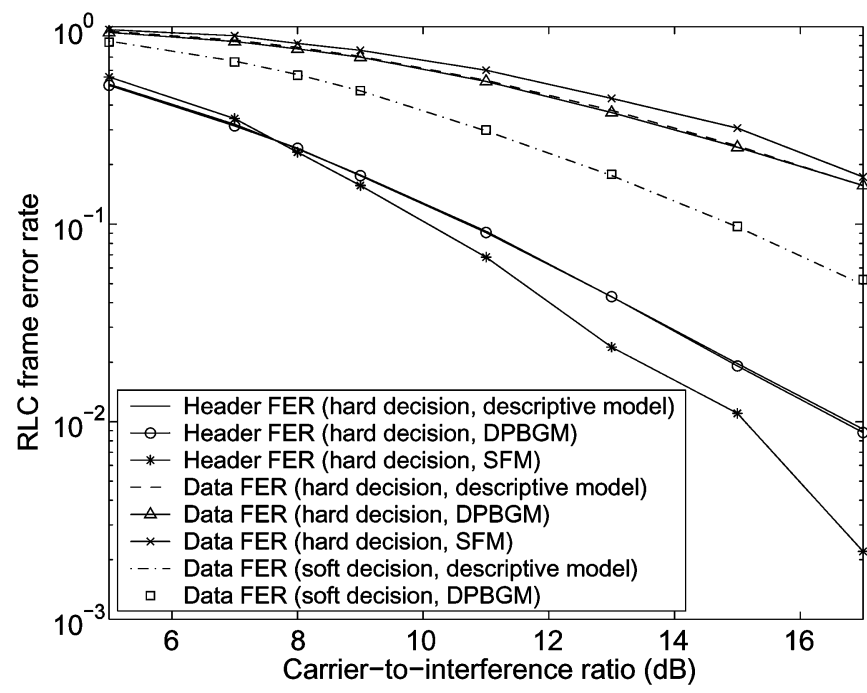

Fig. 9. RLC FERs of the MCS3-coded EGPRS system with hard and soft decoding algorithms obtained from the descriptive models and the generative models.

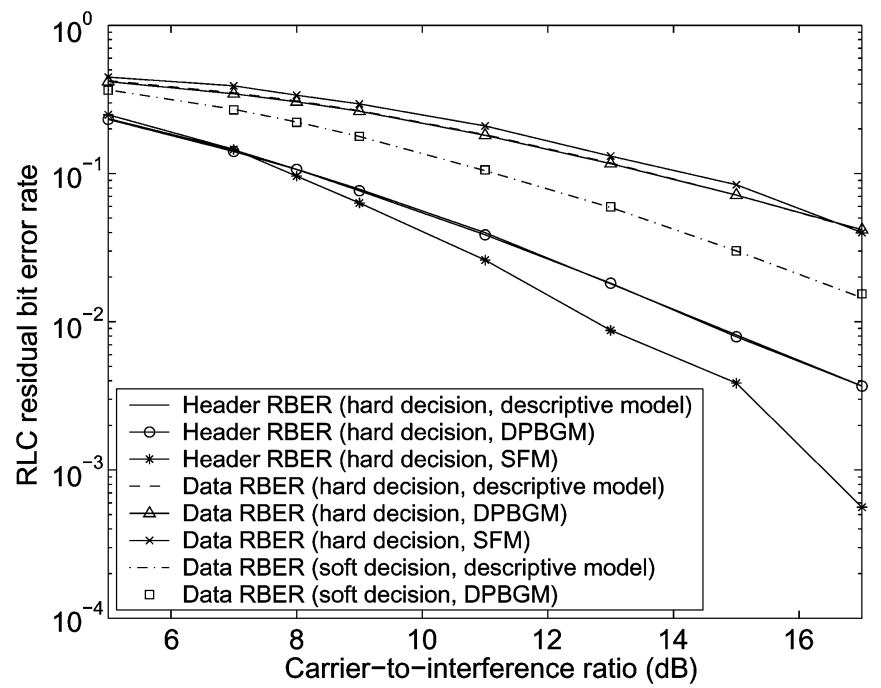

Fig. 10. RLC RBERs of the MCS3-coded EGPRS system with hard and soft decoding algorithms obtained from the descriptive models and the generative models.

\section{APPlication to ERror-CONTROL Strategies}

To further illustrate the accuracy of the proposed DPBGMs, we applied them to the performance evaluation of coded EGPRS systems with hard and soft decoding algorithms. The modulation and coding scheme 3 (MCS3) [35] was chosen as a practical example. Again, only the simulation results for the TU3 IFH channel profile are presented here.

Fig. 9 plots the resulting radio link control (RLC) header and data FERs of the MCS3-coded EGPRS system with a hard decoding algorithm obtained from the descriptive model and two hard generative models. Here, one frame includes four TDMA bursts. It is clear that the FERs obtained from the DPBGM coincide very well with those obtained from the descriptive model, while the accuracy of the FERs obtained from the SFM is not acceptable. The same conclusion holds for the RLC header and data RBERs of the MCS3-coded EGPRS system with a hard decoding algorithm, which are demonstrated in Fig. 10. The RBER is the ratio of the number of errors detected over the frames defined as "good" to the number of transmitted bits in the "good" frames [35].

Figs. 9 and 10 also illustrate the excellent accordance of the resulting RLC data FERs and RLC data RBERs, respectively, of the MCS3-coded EGPRS system with a soft decoding algorithm obtained from the descriptive model and the DPBGM. Good agreements were also observed concerning the corresponding RLC header FERs and RLC header RBERs. We omit the results here in order to retain the clarity of the figures. Obviously, compared with using a hard decoding algorithm, better performance of the MCS3-coded EGPRS system can be obtained by using a soft decoding algorithm.

It is important to mention that we have also successfully applied the proposed DPBGMs to the EGPRS systems with the TU3 NFH, TU50 NFH, and RA275 NFH channel profiles. Furthermore, performance simulations of the coded EGPRS systems with the MCS1 [35] were carried out. Satisfactory results were found in all tested cases. In this manner, the reliability and generality of the proposed DPBGMs, as well as their applicability to coding system evaluation, are validated.

\section{CONCLUSION}

This paper has demonstrated a general procedure of designing a new class of hard and soft generative models by using a properly parameterized and sampled deterministic process followed by a threshold detector and two parallel mappers. Simulation results indicate that the proposed DPBGMs have the attractive capability to approximate very well all the interested burst-error statistics of the underlying descriptive models. The reliability of the suggested hard (soft) DPBGM is further confirmed by performance simulations of coded EGPRS systems with a hard (soft) decoding algorithm obtained from the target and generated hard (soft) error sequences.

\section{REFERENCES}

[1] W. C. Jakes, Ed., Microwave Mobile Communications. Piscataway, NJ: IEEE Press, 1994

[2] P. M. Crespo, R. M. Pelz, and J. Cosmas, "Channel error profiles for DECT," Proc. IEE Commun., vol. 141, no. 6, pp. 413-420, Dec. 1994.

[3] L. N. Kanal and A. R. K. Sastry, "Models for channels with memory and their applications to error control," Proc. IEEE, vol. 66, no. 7, pp. 724-744, Jul. 1977.

[4] M. Zorzi, R. R. Rao, and L. B. Milstein, "ARQ error control for fading mobile radio channels," IEEE Trans. Veh. Technol., vol. 46, no. 2, pp. 445-455, May 1997.

[5] M. Zorzi and R. R. Rao, "Perspectives on the impact of error statistics on protocols for wireless networks," IEEE Pers. Commun., vol. 6, no. 10, pp. 32-40, Oct. 1999.

[6] E. N. Gilbert, "Capacity of a burst-noise channel," Bell Syst. Tech. J., vol. 39, pp. 1253-1265, Sep. 1960.

[7] E. O. Elliot, "Estimates of error rates for codes on burst-noise channels," Bell Syst. Tech. J., vol. 42, pp. 1977-1997, Sep. 1963.

[8] B. D. Fritchman, "A binary channel characterization using partitioned Markov chains," IEEE Trans. Inf. Theory, vol. IT-13, no. 2, pp. 221-227, Apr. 1967.

[9] S. Tsai, "Markov characterization of the HF channel," IEEE Trans. Commun. Technol., vol. COM-17, no. 1, pp. 24-32, Feb. 1969.

[10] F. Swarts and H. C. Ferreira, "Markov characterization of digital fading mobile VHF channels," IEEE Trans. Veh. Technol., vol. 43, no. 4, pp. 977-985, Nov. 1994.

[11] A. Semmar, M. Lecours, J. Y. Chouinard, and J. Ahern, "Characterization of error sequences in UHF digital mobile radio channels," IEEE Trans. Veh. Technol., vol. 40, no. 4, pp. 769-776, Nov. 1991.

[12] J. Garcia-Frias and P. M. Crespo, "Hidden Markov models for burst error characterization in indoor radio channels," IEEE Trans. Veh. Technol., vol. 46, no. 6, pp. 1006-1020, Nov. 1997. 
[13] W. Zhu and J. Garcia-Frias, "Stochastic context-free grammars and hidden Markov models for modeling of bursty channels," IEEE Trans. Veh. Technol., vol. 53, no. 3, pp. 666-676, May 2004.

[14] A. Willig, "A new class of packet- and bit-level models for wireless channels," in Proc. IEEE PIMRC, Lisbon, Portugal, Sep. 2002, pp. 2434-2440.

[15] W. Turin, Digital Transmission Systems: Performance Analysis and Modeling. New York: McGraw-Hill, 1999.

[16] E. Costamagna, L. Favalli, and P. Gamba, "Multipath channel modeling with chaotic attractors," Proc. IEEE, vol. 90, no. 5, pp. 842-859, May 2002.

[17] E. Costamagna, L. Favalli, P. Gamba, and P. Savazzi, "Block-error probabilities for mobile radio channels derived from chaos equations," IEEE Commun. Lett., vol. 3, no. 3, pp. 66-68, Mar. 1999.

[18] E. Costamagna, L. Favalli, P. Savazzi, and F. Tarantola, "Long sequences of error gaps derived from chaotic generators optimized for short ones in mobile radio channels," in Proc. IEEE VTC, Los Angeles, CA, Sep. 2004, pp. 4250-4254.

[19] A. Köpke, A. Willig, and H. Karl, "Chaotic maps as parsimonious bit error models of wireless channels," in Proc. IEEE INFOCOM, San Francisco, CA, Mar. 30-Apr. 3, 2003, pp. 513-523.

[20] C. X. Wang and M. Pätzold, "A novel generative model for burst error characterization in Rayleigh fading channels," in Proc. IEEE PIMRC, Beijing, China, Sep. 2003, pp. 960-964.

[21] _ - "A generative deterministic model for digital mobile fading channels," IEEE Commun. Lett., vol. 8, no. 4, pp. 223-225, Apr. 2004

[22] — " "Deterministic modeling and simulation of error sequences in digital mobile fading channels," in Proc. IEEE ICC, Paris, France, Jun. 2004, pp. 3374-3378.

[23] _ _ "A new deterministic process based generative model for characterizing bursty error sequences," in Proc. IEEE PIMRC, Barcelona, Spain, Sep. 2004, pp. 2134-2139.

[24] F. Swarts and H. C. Ferreira, "Markov characterization of channels with soft decision outputs," IEEE Trans. Commun., vol. 41, no. 5, pp. 678-682, May 1993.

[25] N. Nefedov, "Generative Markov models for discrete channel modelling," in Proc. IEEE PIMRC, Helsinki, Finland, Sep. 1997, pp. 7-11.

[26] - "Realistic discrete channel models for error control strategies evaluation," in Proc. SBT/IEEE ITS, Sao Paulo, Brazil, Aug. 1998, pp. $37-42$.

[27] gies in CDMA," in Proc. IEEE ISSSTA, Sun City, South Africa, Sep. 1998, pp. 245-249.

[28] - "Discrete channel models for wireless data services," in Proc. IEEE VTC, Ottawa, ON, Canada, May 1998, pp. 683-687.

[29] E. Costamagna, L. Favalli, P. Savazzi, and F. Tarantola, "Modeling of wireless channel behavior for fast simulations: A soft-bit level perspective," in Proc. IEEE VTC, Oct. 2003, pp. 173-177.

[30] , "Evaluation of soft-bit error sequence generators at the output of the decoding process," in Proc. IEEE VTC, Milan, Italy, May 2004, pp. $1028-1032$.

[31] S. O. Rice, "Mathematical analysis of random noise," Bell Syst. Tech. J., vol. 23, pp. 282-332, Jul. 1944.

[32] - "Mathematical analysis of random noise," Bell Syst. Tech. J., vol. 24, pp. 46-156, Jan. 1945.

[33] M. Pätzold, Mobile Fading Channels. New York: Wiley, 2002.

[34] C. X. Wang, M. Pätzold, and B. Itsarachai, "A deterministic frequency hopping Rayleigh fading channel simulator designed by using optimization techniques," in Proc. IEEE PIMRC, Lisbon, Portugal, Sep. 2002, pp. 478-483.

[35] Radio Transmission and Reception (Rel. 4), 3GPP TS 45.005, 3GPP, 2003.

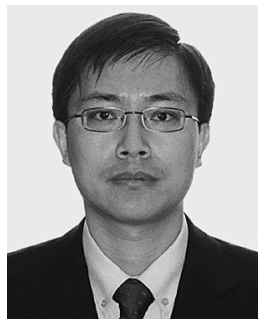

Cheng-Xiang Wang (S'01-M'05) received the B.Sc. and M.Eng. degrees in communication and information systems from Shandong University, Shandong, China, in 1997 and 2000, respectively, and the $\mathrm{Ph} . \mathrm{D}$. degree in wireless communications from Aalborg University, Aalborg, Denmark, in 2004.

From 2000 to 2001, he was a Research Assistant with the Department of Communication Networks, Technical University of Hamburg-Harburg, Hamburg, Germany. From 2001 to 2005, he was a Research Fellow of mobile communications with Agder University College, Grimstad, Norway. From January to April 2004, he was a Visiting Researcher at the Baseband Algorithms and Standardization Laboratory, Siemens AG-Mobile Phones, Munich, Germany, conducting research and development of error models for EGPRS systems within the framework of the 3GPP GERAN System Concept R\&D Project. Since 2005, he has been a Lecturer in Electrical, Electronic and Computer Engineering, School of EPS, Heriot-Watt University, Edinburgh, U.K. Since June 2006, he has been a Honorary Fellow of the School of Engineering and Electronics, University of Edinburgh, and an Adjunct Professor of Guilin University of Electronic Technology, China. His current research interests include mobile radio channel modeling, error models, smart antennas and MIMO systems, OFDM, UWB, cognitive radio, cross-layer design of wireless networks, mobile ad hoc and wireless sensor networks, and 3GPP evolved UTRA(N). He has published about 70 research papers in journals and conference proceedings.

Dr. Wang was the recipient of the 2001 Excellent Master Thesis Award of Shandong Province. Also, he received the 1999 Excellent Paper Award for two of his papers from the sixth National Youth Communication Conference of China, Beijing, China. He serves as an Editorial Board member for the Wireless Communications and Mobile Computing Journal and is a TPC member for 14 major international conferences, including IEEE VTC 2005-Fall, ICIC 2006, Globecom 2006, WCNC 2007, ICIC 2007, and ICC 2007. He also served as Session Chair for ICCCAS 2006. He is a member of the IET.

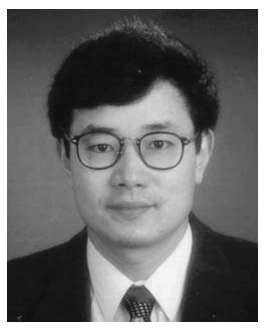

Wen Xu (M'96-SM'03) received the B.Sc. degree in 1982 and the M.Sc. degree in 1985 from Dalian University of Technology (DUT), Dalian, China, and the Dr.-Ing. (Ph.D.) degree in 1996 from Munich University of Technology (TUM), Munich, Germany, all in electrical engineering.

Since 1995, he has been with Siemens AG-Mobile Phones (now BenQ Mobile), Munich, Germany, where he is responsible for several $R \& D$ projects and has actively participated and contributed to standardization activities of ETSI and 3GPP. Since 2000, he has been the Head of the Baseband Algorithms and Standardization Laboratory, which is responsible for physical layer and multimedia signal processing, and protocol stack aspects. As a competence center, his laboratory has been involved in different standardization activities such as 3GPP and DVB for 2G, 3G, beyond 3G mobile systems, as well as the DVB-H system. He also served as a consultant of Department of Electronic Engineering of DUT. His research interests include image/video/speech coding and processing, channel coding, equalization, cross-layer system design, and mobile communications.

Dr. Xu is a member of the Verband der Elektrotechnik, Elektronik, Informationstechnik (VDE), Germany. 